\title{
The Development of a Cryogenic Over-Pressure Pump
}

\author{
M. Alvarez*, H. Cease*, B. Flaugher*, R. Flores*, J. Garcia†, A. Lathrop* and F. \\ Ruiz $^{\dagger}$ \\ *Fermi National Accelerator Laboratory, Batavia, IL, 60510 \\ ${ }^{\dagger}$ Illinois Institute of Technology, Chicago, IL, 60616
}

\begin{abstract}
A cryogenic over-pressure pump (OPP) was tested in the prototype telescope liquid nitrogen (LN2) cooling system for the Dark Energy Survey (DES) Project. This OPP consists of a process cylinder (PC), gas generator, and solenoid operated valves (SOVs). It is a positive displacement pump that provided intermittent liquid nitrogen (LN2) flow to an array of charge couple devices (CCDs) for the prototype Dark Energy Camera (DECam). In theory, a heater submerged in liquid would generate the drive gas in a closed loop cooling system. The drive gas would be injected into the PC to displace that liquid volume. However, due to limitations of the prototype closed loop nitrogen system (CCD cooling system) for DECam, a quasiclosed-loop nitrogen system was created. During the test of the OPP, the CCD array was cooled to its designed set point temperature of $173 \mathrm{~K}$. It was maintained at that temperature via electrical heaters. The performance of the OPP was captured in pressure, temperature, and flow rate in the CCD LN2 cooling system at Fermi National Accelerator Laboratory (FNAL).
\end{abstract}

Keywords: pump, closed loop system, DES, Dark Energy Survey Project, telescope PACS: $07.20 . \mathrm{Mc}$

\section{INTRODUCTION}

The over-pressure pump (OPP) is a positive displacement (PD) pump that utilizes the gaseous phase of a fluid to displace a liquid volume within a piston cylinder. It utilizes the work available from the large volume expansion ratio of a liquid to gas. For instance, a cubic centimeter of liquid nitrogen (LN2), at atmospheric conditions can expand about 700 times that volume into nitrogen gas (GN2). This work is available by using a heating element submerged in a liquid volume, which is used to generate a gas volume. That gas volume or drive gas is injected into a process cylinder (PC), which encases the liquid volume. As pressure builds within the PC, the liquid is displaced out to the heat exchanger.

An experiment was conducted to test the feasibility of this pumping concept. A prototype cooling system for the Dark Energy Survey (DES) Project [7] was used to test this device and other prototypes. The primary system is a closed loop liquid nitrogen system (CCD cooling system) located on the Blanco 4m Telescope at Cerro Tololo InterAmerican Observatory in Chile. It is used to cool and maintain the temperature of the charged couple device (CCD) to $173 \mathrm{~K}$ [5]. One cryogenic refrigerator is capable of removing $360 \mathrm{~W}$ from the system. Additional system specifications can be found in Cooling the Dark Energy Camera CCD array using a closed-loop, two-phase liquid nitrogen system [5].

\section{DESCRIPTION OF THE OVER-PRESSURE PUMP}

The cryogenic OPP was used to cool CCDs in a secondary or prototype system. Its primary goal was to cool an array of 74 charge coupled devices (CCDs) to a set point temperature of 173K. It had done so using the concept of a positive displacement direct acting piston type pump [6]. These particular devices use a drive gas to displace a liquid volume. This function is performed by a piston and a piston rod that actuate in and out of a cylinder in a reciprocating fashion [6]. The OPP eliminates the piston and piston rod and replaces it with a gas-liquid moving interface.

Evidence of the OPP operating was captured in flow rate, temperature, and pressure measurements. This device, in theory, consists of a process cylinder (PC), gas generator, and solenoid operated valves (SOVs) [3]. The PC stores the coolant. The gas generator stores liquid similarly to the PC, but contains a liquid submerged heater to generate the drive gas. This device provides the drive fluid that is injected into the PC to displace the liquid volume. The SOVs control the refill, discharge, and delay of the PC. 
The OPP operated in a quasi-closed loop CCD cooling system at Fermi National Accelerator Laboratory (FNAL). This system was created as a result of a tests conducted in December 2011 [1], which indicated that the OPP could not sufficiently pump quickly enough to maintain the array of CCDs at $173 \mathrm{~K}$. This cooling system contains one $360 \mathrm{~W}$ cryogenic refrigerator. It is capable of condensing GN2 at 7LPH (LN2) [5]. In the quasi-closed loop cooling system, the high system pressure, after each pumping sequence of the PC, was vented to atmosphere. The primary components in the system consist of the PC, SOVs, and a modified nitrogen gas (GN2) generator.

\section{EXPERIMENTAL SETUP}

A test of the SOVs and PC was conducted in Lab A at FNAL. It was completed over a three day period in February 2012. The primary goal of the test is to determine whether the SOVs and PC was capable of cooling the multi-CCD (MCCD) test vessel focal plate to $173 \mathrm{~K}$. Completing this objective would indicate that the SOVs were controlling the pumping and refilling of the PC effectively. The MCCD vessel is stationed on a cart that is on ground level. It contains a tube heat exchanger, in a vertical position that attaches copper braids to and from the focal plate. Each copper braid contains an electrical heater to regulate the temperature to $173 \mathrm{~K} \pm 0.25 \mathrm{~K}$ [5].The vacuum insulated supply and return transfer lines are routed from the 200L cryogenic liquid vessel (CLV) outside Lab A to the MCCD test vessel.

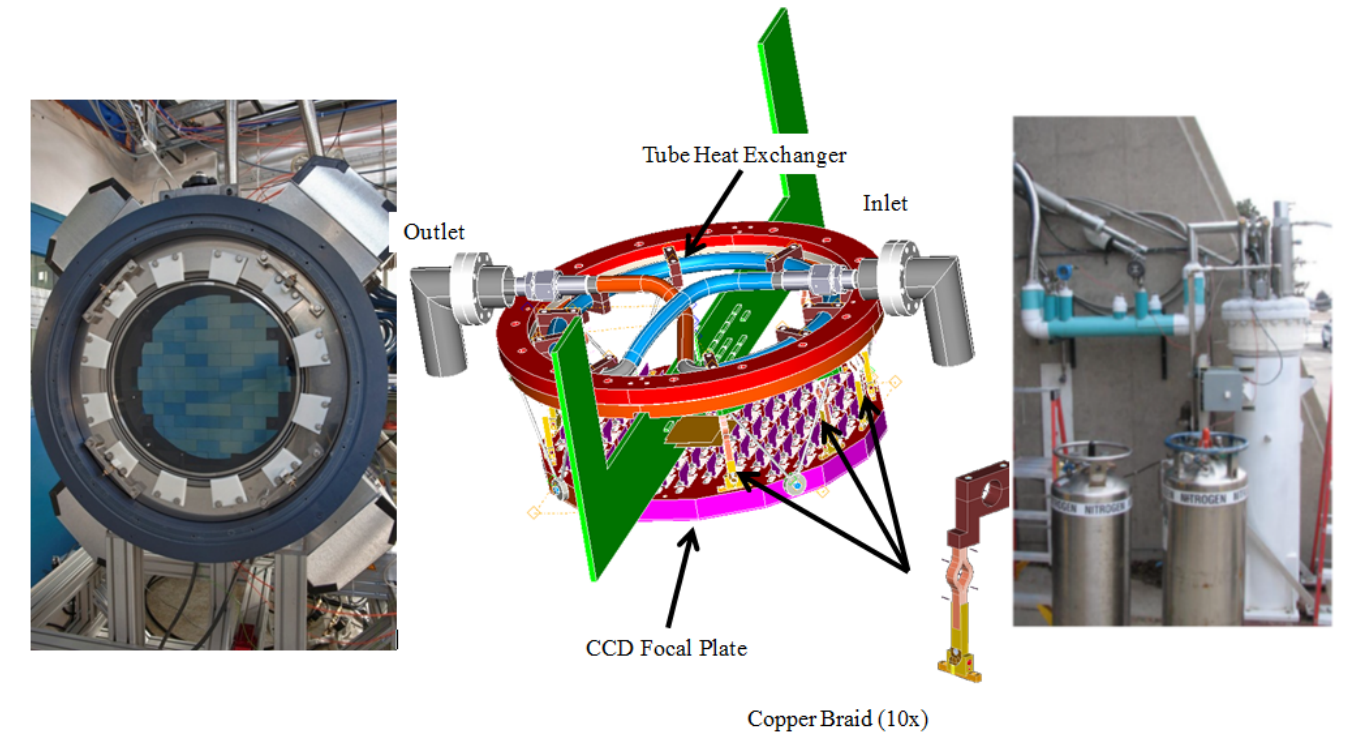

FIGURE 1. MCCD Test Vessel (left), CCD Focal Plate and Tube Heat Exchanger (middle), 200L Cryogenic Liquid Vessel (right)

The tube heat exchanger that regulates the temperature of the focal plate can be seen in Figure 1 (middle). Each copper braid contains a 20W heater [5]. Various resistance temperature detectors (RTDs) were installed along the MCCD focal plate and throughout the CCD cooling system. Additionally, pressure, flow rate, and liquid level instrumentation was installed within the system. The data from these devices was recorded using LabView 2010 V10.0 (32-bit) [2].

The quasi-closed loop cooling system (QCLCS) was created so that the PC can replenish its liquid volume and supply LN2 to the CCD focal plate. The closed loop portion of the cooling system occurs when LN2 is being pumped. The open loop portion occurs when LN2 refills the PC. A vent (SOV-004) on the drive gas supply line (GN2 Injection Line) was added to relieve the pressure within the system. This allowed the PC to use the difference between the hydrostatic pressure and atmospheric pressure to induce flow into the PC to refill it with LN2. Three SOVs, SOV-002, SOV-003, and SOV-004, were used to control this refill process. Additionally, the GN2 generator is replaced with a 160L low pressure cryogenic liquid vessel, which has a supply pressure of 22 psig or 1.51 bar. The liquid from the dewar was supplied to the one quarter inch copper tubing GN2 injection line (see Fig. 2), which was uninsulated copper tubing. This setup was meant to vaporize the liquid volume to generate the drive gas [4]. The next set of diagrams show a schematic of the pumping and refilling schemes of the quasi-closed loop cooling system. 


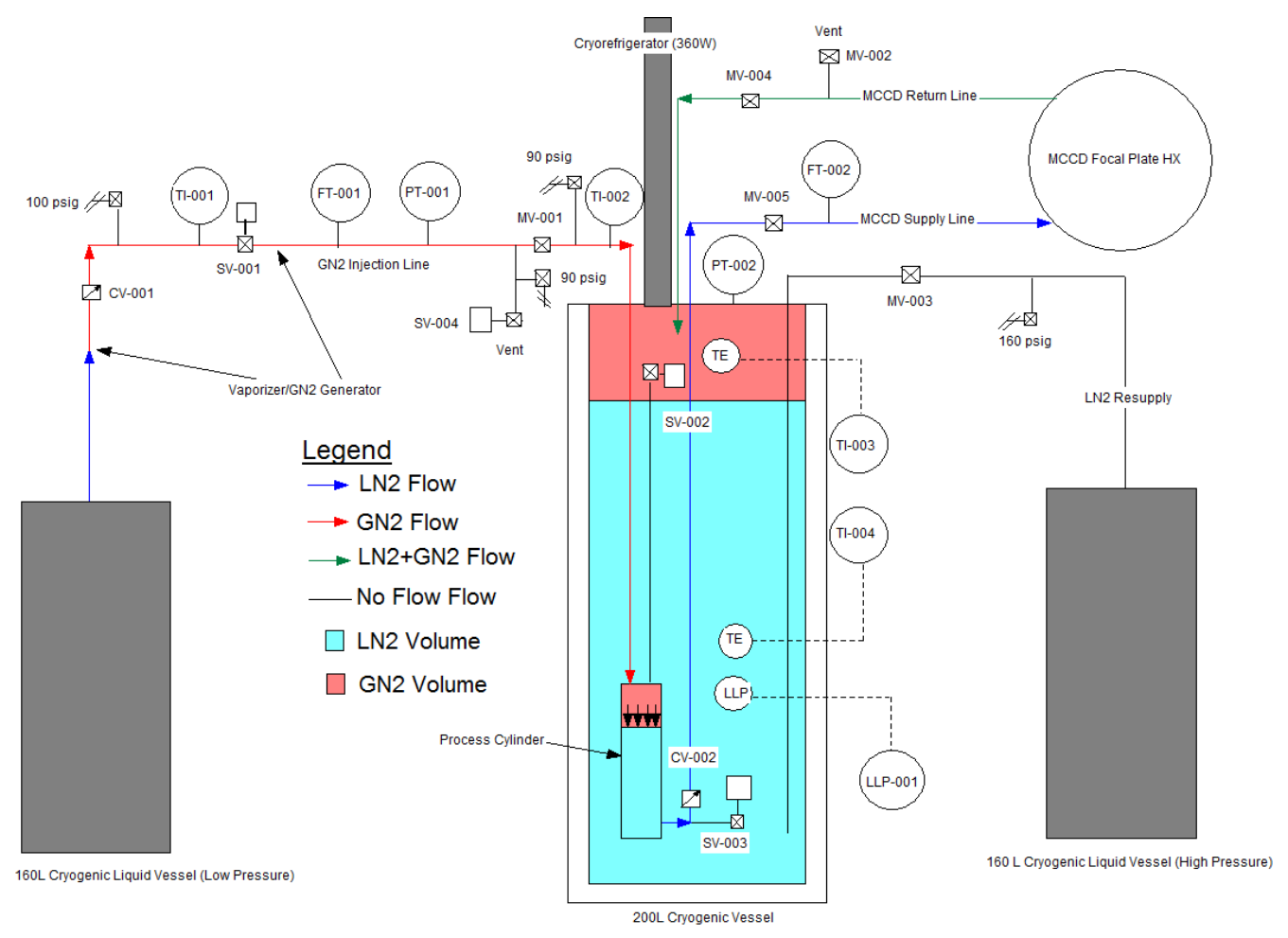

FIGURE 2. Pumping or Discharging LN2 from the PC

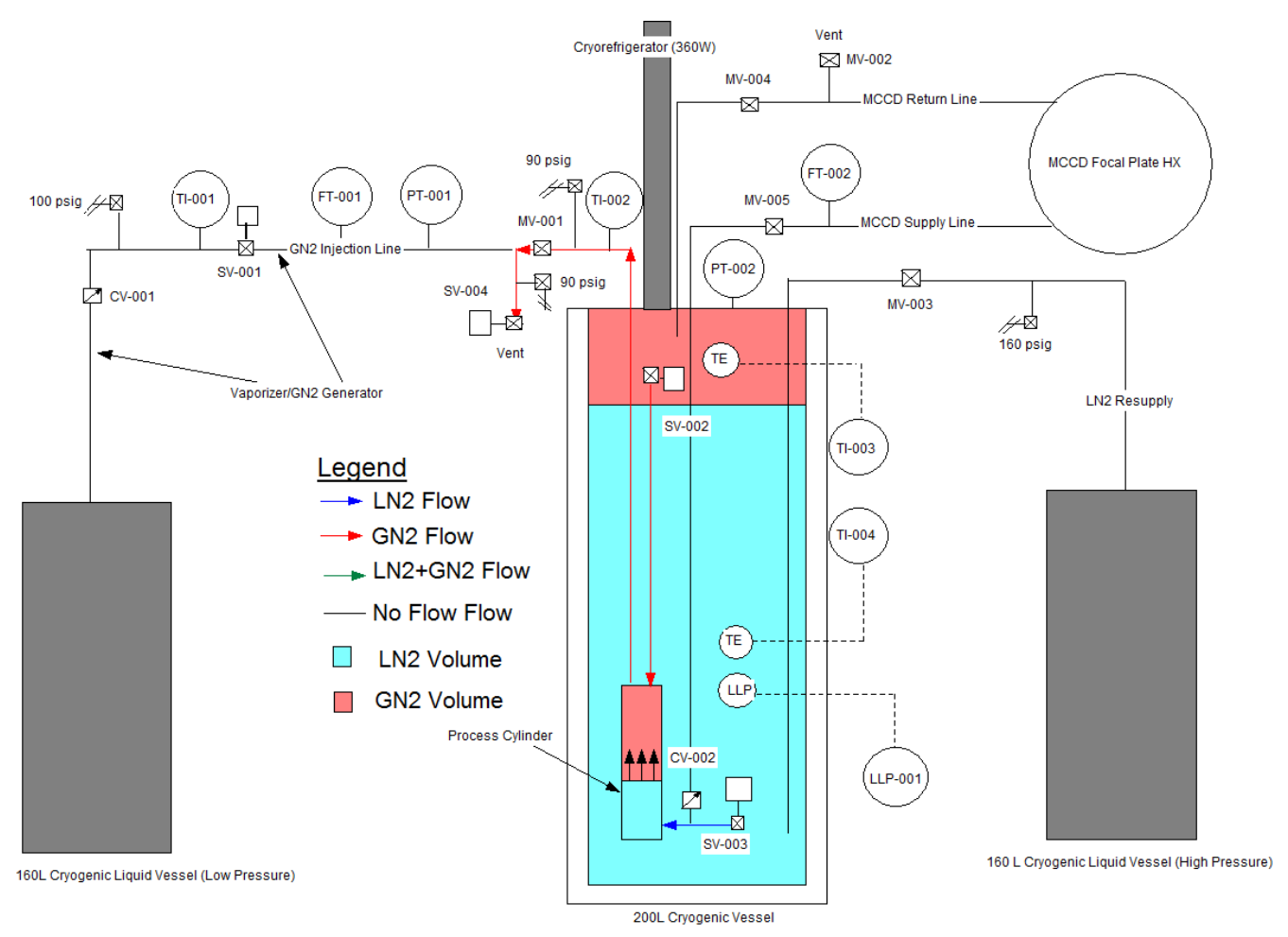

FIGURE 3. Refilling of the PC 
The pumping and refilling operations, which are controlled by the SOVs are seen in Figures 2 and 3 . Cold GN2 $(185 \mathrm{~K})$ is injected into the PC for 40 s and the volume of LN2 is displaced outwards towards the MCCD focal plate tube heat exchanger as seen in Figure 2. After the 40s pumping interval, SOV-002, SOV-003, and SOV-004 open and SOV-001 closes to allow the fully submerged PC to fill as in Figure 3 (bottom). LN2 enters through SOV-003 and warm GN2 exits through SOV-004, which lowers the system pressure. Venting lowers the system pressure to allow the hydrostatic pressure of the LN2 column height, within the 200L CLV, to refill the PC. Refilling of the PC lasts for 100s. A 15s delay was added to mark each consecutive cycle of the OPP [3]. The general timing sequence used for this cryogenic OPP is $15 \mathrm{~s}, 40 \mathrm{~s}$, and 100s for a delay, discharge, and refill. Two other data sets with different refill times (60s and 100s) have been collected and are stored in Dark Energy Document Database [1][3].

The objectives of the PC test were the following.

1. Cool the focal plate to $173 \mathrm{~K}$ using the quasi-closed loop CCD cooling system.

2. Attain a correlation of the average flow rates of the GN2 drive fluid (injection) and LN2 cooling supply (discharge) of the PC.

$$
\dot{V}_{G N 2, \text { drivegas }}=\dot{V}\left(\dot{V}_{L N 2, \text { supply }}\right)
$$

3. Determine the number of cryogenic refrigerators needed to operate the system closed loop.

\section{DATA}

The data described in this section is specific to refill time of 100s. There are three parts of a single 155s pumping cycle for the cryogenic over-pressure pump test. The timing sequence is a delay, discharge, and refill [1]. A 15s delay is meant to discern from each consecutive cycle. The delay is done by closing all solenoid actuated valves within the system (see Figs. 2 and 3). The next part of the cycle is a discharge. A discharge refers physically displacing LN2 from the PC. Lastly, a refill refers to filling the PC with LN2 by utilizing the hydrostatic pressure of the LN2 level within 200L CLV, which is typically about 0.75 psi or 0.05 bar.
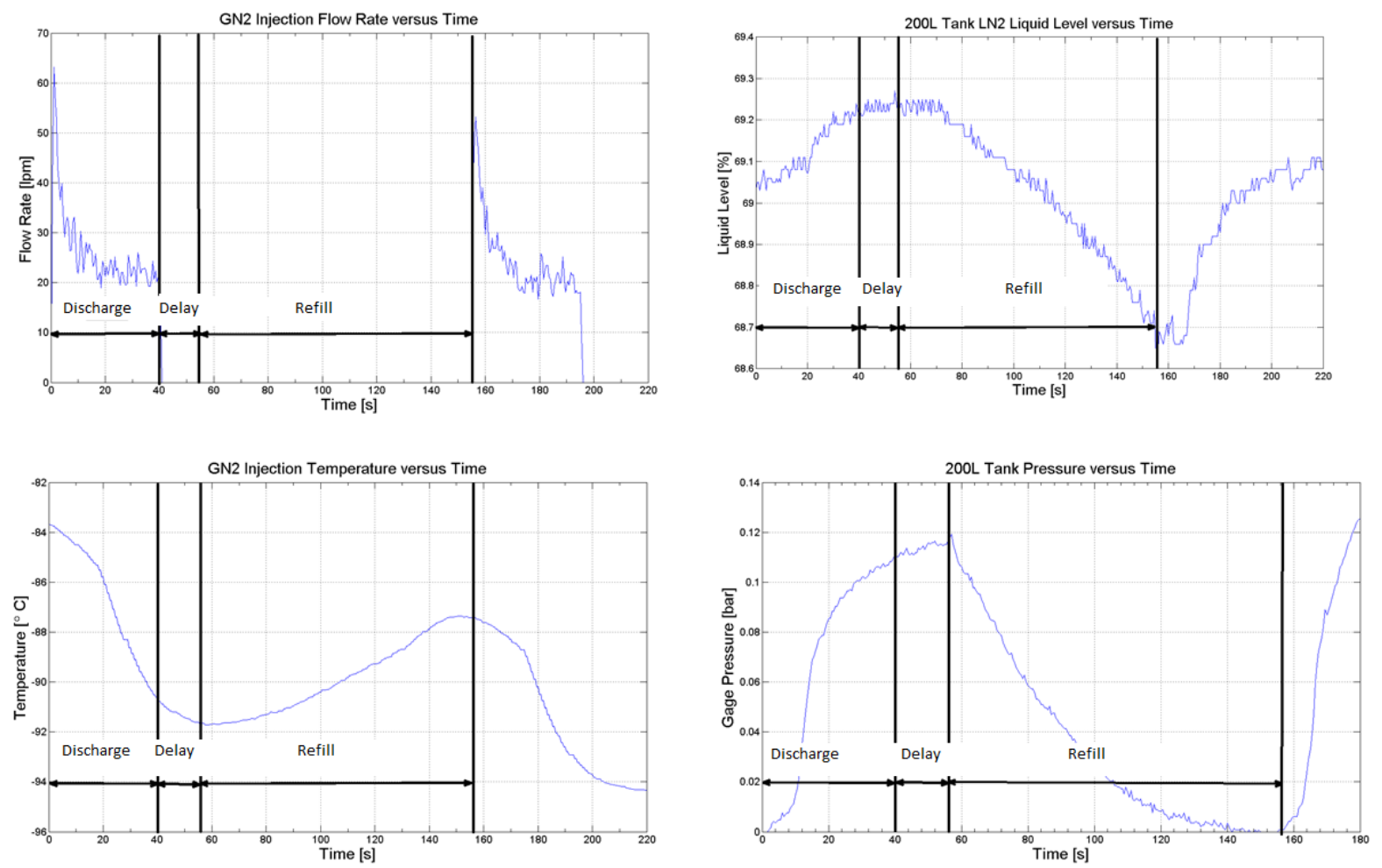

FIGURE 4. GN2 Injection Flow Rate: FT-001 (top left). Liquid Level Probe for 200L CLV: LLP-001 (top right). Nitrogen Drive Gas Temperature: TI-001(bottom left). 200L CLV Pressure: PT-002(bottom right).) 
Vertical black lines discern between a discharge, delay, and refill of the OPP cycle for instruments FT-001, LLP001, TI-001, and PT-002 (see Fig. 4). The figures above represent only the data sets associated with refill time of 100s. FT-001 indicates that the GN2 is flowing. LLP-001 shows that the dynamics of the liquid level within the 200L CLV during the cycle timing sequence. TI-001 shows how the drive fluid temperature, GN2, changes with time during each part of the cycle.

The gas injection flow rate only lasts 40s (Fig. 4 (top left)). The flow rate begins high and decays to a constant value. This is a result of the differential pressure between the $200 \mathrm{~L}$ and the $160 \mathrm{~L}$ lower pressure CLVs approaching a constant value. The pumping process continues until the delay portion of the cycle begins and closes all SOVs (SV001 to SV-004), which corresponds to zero flow rate in the GN2 injection line. After 15s, the PC refill is initiated by opening SOV-002 to SOV-004 (refer to Figure 3) and closing SOV-001 [3].

After 40s of pumping on the PC, an increase in the LN2 level of the 200L CLV is observed in Figure 4 (top right). This corresponds to LN2 flow traversing through the MCCD transfer lines and returning to the 200L CLV as seen in Figure 2. A delay shows a plateau or constant liquid level, which corresponds to no liquid displacement. Finally, the tank refill shows a decrease in liquid level, which corresponds to LN2 refilling the process cylinder volume due to the hydrostatic pressure [3].

Notice that during the discharge of the PC, the temperature (TI-001 in Fig. 2) of the GN2 injection line becomes colder as seen in Figure 4 (bottom left). This is associated with the cold GN2 gas flowing through the cyclically warming line; the line warms as result of radiation, convection, and conduction heat transfer from the environment after each cycle. Furthermore, the delay is associated with the beginning of a warming trend, which is a result of the discontinuance of GN2 flow by closing SOV-001. Because flow in the GN2 injection line ceases, radiation heat transfer begins to dominate, which causes the line to warm [3].

The pressure increases within the 200L CLV during the 40s GN2 injection process (see Fig. 4 (bottom right)). Notice that the pressure within the 200L cryogenic liquid vessel (CLV) increases during the GN2 injection process. This a result of the system being closed loop during the discharge portion of the cycle or when LN2 is supplied to the tube heat exchanger. During the delay, notice that a slight increase in pressure can be seen as a result of the environmental heat load, which increases the pressure of the system by boiling LN2. The tank refill shows a drop in pressure because SOV-002 to SOV-004 are open to vent the high pressure GN2 to atmosphere [3].

\section{RESULTS}

Once the data was collected from the pressure transducers, liquid level probe, flow meters, and RTDs, it was converted to the International System of Units (SI). The focal plate was cooled to $173 \mathrm{~K}$. A plot depicting the mass and volume flow rates of the gas injected to the liquid displaced was created using data from different refill times $(60 \mathrm{~s}$, 100s, and 120s) [3]. Lastly, the number of cryogenic refrigerators was estimated. Figure 5 shows the average focal plate temperature versus time.

The ascending temperature of the focal plate indicate that the trim heaters on the copper braids are turned on (see Fig. 5). These heaters are meant to regulate the focal plate temperature to in and around 173K [5]. Furthermore, notice that in Figure 5, the temperature of the injected GN2 (see Fig. 4 (TI-001) is significantly above 173K (see Fig. 4 (bottom left). This implies that the GN2 injected into the PC cannot be the reason the focal plate cooling because the vaporizer was not operating correctly. This warming trend indicates that LN2 is flowing through the MCCD transfer lines to the tube heat exchanger cooling the focal plate to below 173K. Lastly, notice that the RTDs show different temperature measurements. This is a result of imperfect contact between the copper braids and the heat exchanger tubing (see Fig. 1). Hence, the difference in temperature of RTD10 and RTD2 in the Figure 5.

Inlet and outlet mass and volume flow rate plots consist of three points, which represent refill times of 60s, 100s, and 120s (see Fig. 6) [1][3]. The refill time corresponds to the time allotted for LN2 to enter the PC. For example, a refill time of 60 s will result in the PC having less LN2 volume available for cooling the CCDs than for a refill time of 100s [3]. On the contrary, their is a limit at which the volume of LN2 approaches a maximum. This limit is governed by the maximum fill of LN2 within the PC, which is controlled by the orifice diameter of SOV-003 line (see Fig. 3) and the hydrostatic pressure of the LN2. The limit is $2.5 \mathrm{ft}$ of LN2 hydraulic head above the SOV-003 line.

The volumetric flow rate correlation can be used to extrapolate LN2 flow rates within the 60s to 100 s refill time range as seen in Figure 6. These correlations provide a way of estimating the amount of GN2 that is needed to displace a fixed amount of LN2 from the PC. Notice that their is abrupt rise in flow rate between refill times $60 \mathrm{~s}$ and $100 \mathrm{~s}$ in Figure 6. This abrupt change is directly associated with the increase in the time to refill the process cylinder (PC). However, their seems to be almost no increase in LN2 flow rate from refill times 100s to 120s. This is associated 


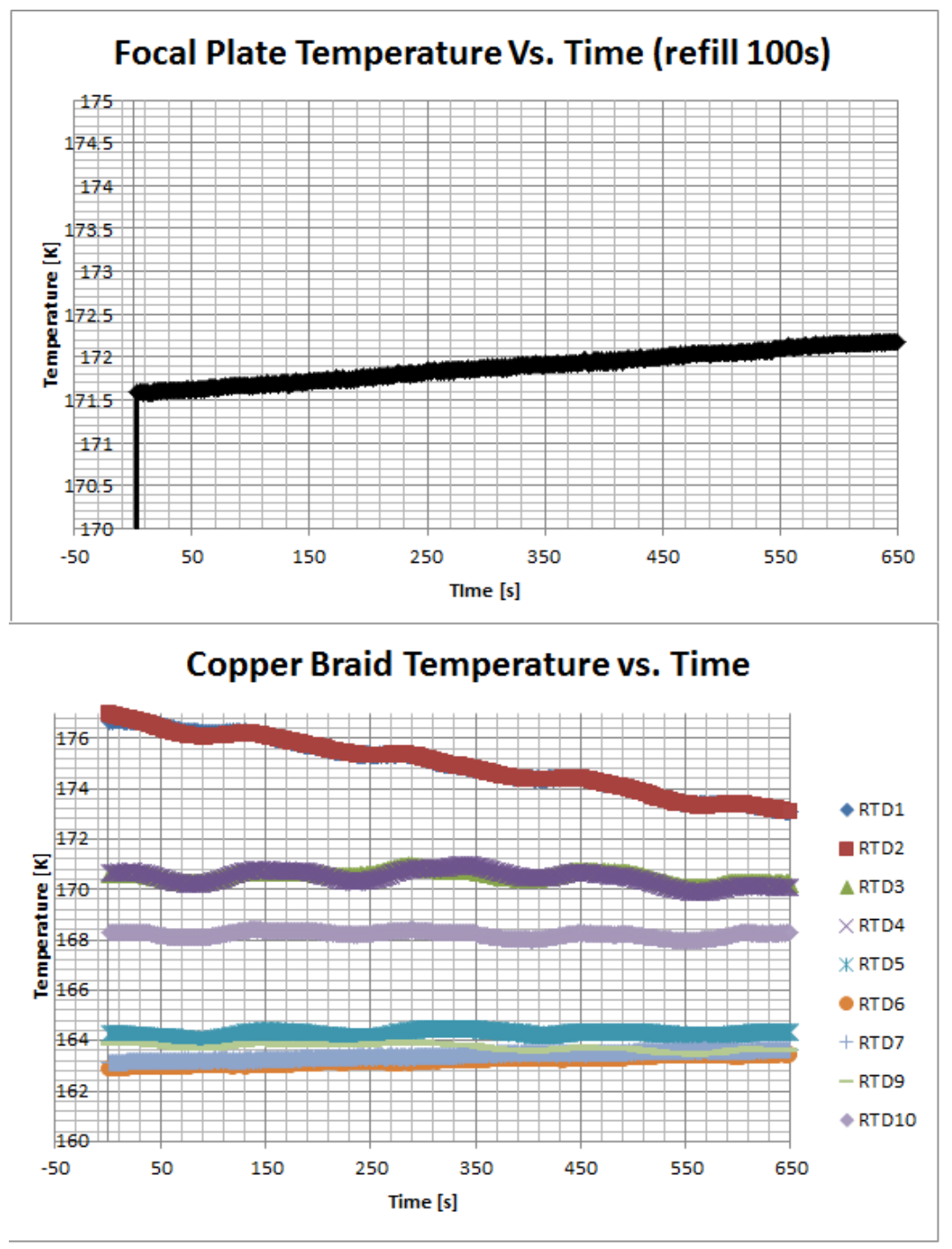

FIGURE 5. Focal Plate Temperature vs. Time (top). Copper Braid Temperature vs. Time (refill 100s) (bottom)

with the suction end of the process cylinder. In other words, the orifice size of the PC restricts it from refilling via hydrostatic pressure. Notice the line intersecting the points of refill times 60s and 100s in Figure 6. This line provides evidence that their is a linear relationship between the GN2 injected into the PC and the discharged LN2. This linear relationship provides an operating curve that is specific to the pump's current operating conditions (i.e. heat load on the system, differential pressures, temperatures). Additionally, notice a horizontal black line drawn through refill time of 120s seen in Figure 6. This states that the LN2 flow rate, for any GN2 injection flow rate, will peak at $0.6 \mathrm{~kg} / \mathrm{min}$ or 0.75LPM. Therefore, any additional GN2 drive fluid injected into the PC will have no effect in the LN2 flow rate. In other words, this point maximizes the LN2 flow rate and minimizes the GN2 injection flow rate for this specific case.

The last objective involves estimating the number of cryogenic refrigerators to operate the cryogenic OPP in a closed loop CCD cooling system. A mass and energy balance of the system was performed given the pressure, flow rate, and temperature data collected [2]. The total energy from environmental heat loads and the energy associated with the drive gas was found and compared to the $316 \mathrm{~W}$ cryogenic refrigerator used in the experiment. It was discovered that $1305 \mathrm{~W}$ must be removed from the system before the next cycle commenced. Therefore, the total number of cryogenic refrigerators required to operate closed loop, for refill time of $100 \mathrm{~s}$, is 6 . Furthermore, the number of cryogenic refrigerators required for refill time of $60 \mathrm{~s}$ and $120 \mathrm{~s}$ are 5 and 8 . The number of cryogenic refrigerators is directly correlated to the refill time. This is a result of the GN2 injection line, in Figure 2, being a vaporizer. In 


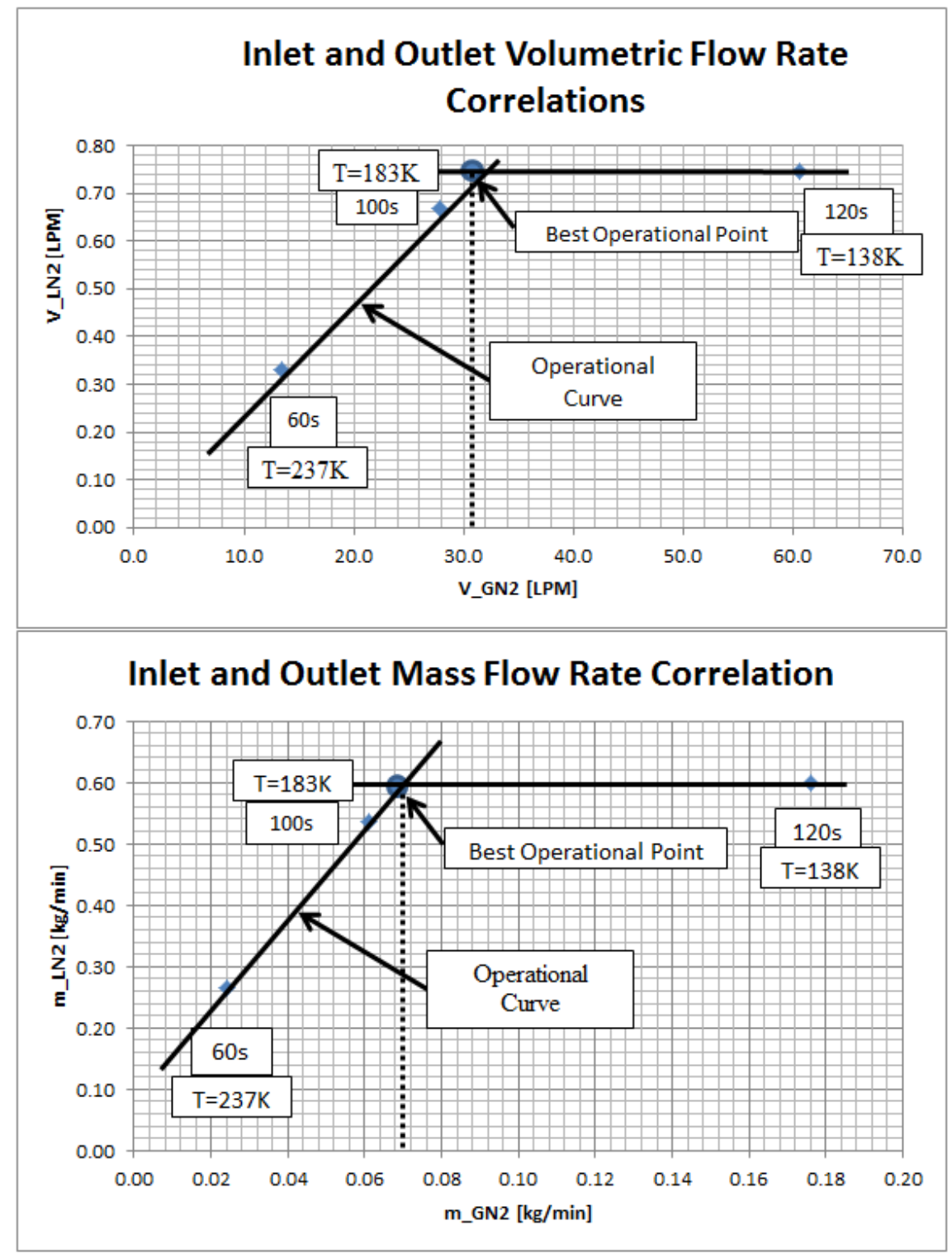

FIGURE 6. Top: Volumetric Flow Rate correlation. Bottom: Mass Flow Rate Correlation

other words, during a refill the GN2 injection upstream of the FT-001 has zero flow. This implies that the uninsulated tubing begins to warm the longer the refill time is. This increases the heat input of the vaporizer. The heat inputs to the vaporizer was estimated for refill times $60 \mathrm{~s}, 100 \mathrm{~s}$, and 120 s to be $1035 \mathrm{~W}, 1305 \mathrm{~W}, 1892 \mathrm{~W}$.

Flow through the MCCD transfer line is captured in four different instruments seen in Figures 4. The trim heaters regulated the focal plate temperature to $173 \mathrm{~K}$. They would turn off and on when the focal plate temperature is above and below 173K. Any subtle thermal disturbances would force the trim heaters to adjust their power states. Thus, as pumping commenced the focal plate became cooler, which would turn the heaters on. Lastly, the RTDs mounted on the copper braids indicate that the LN2 within tube heat exchanger is well below the set point of the focal plate (i.e. 166K, $168 \mathrm{~K}$ ), which means the LN2 supply from the OPP is sufficient for intermittent flow. Lastly, the subtle oscillation seen in Figure 5 (bottom) show the effects of the reciprocating pump.

Three different refill times were attempted: 60s, 100s, and 120s [2][3]. The linear region, seen in Figure 6, show where the maximum LN2 flow rate will occur for minimal drive gas. Recall, that the drive gas must be condensed back into liquid when in a closed loop. Also, notice that the curve no longer becomes linear for a GN2 flow rate greater 
than 30LPM for this particular PC. This is associated with the orifice size near SOV-003 in Figure 3 constricting the flow into the PC. An orifice size greater than $3 / 4$ inch would promote better refills larger than $1 \mathrm{~L}$. Currently, a refill of $0.91 \mathrm{~L}$ has been achieved for a nearly $2.5 \mathrm{ft}(0.75 \mathrm{~m})$ hydraulic head of LN2 [3]. The fill flow rate entering the PC for refill time of 100s was estimated to be 0.55LPM [2]. For instance, increasing the refill time from 60s to $100 \mathrm{~s}$ increases the flow rate and volume into the PC by $0.05 \mathrm{LPM}$ and $0.4 \mathrm{~L}$; ideally, the refill volume should be $0.2 \mathrm{~L}$.

\section{SUMMARY}

Overall, the cryogenic OPP SOVs and PC operated in a quasi-closed loop CCD cooling system at FNAL. The focal plate was cooled to $173 \mathrm{~K}$, an inlet and outlet flow rate correlation was derived, and the number of cryogenic refrigerators was estimated for each refill time. The OPP SOVs and PC functioned and provided intermittent flow, which cooled the focal plate to $173 \mathrm{~K}$. Evidence of the OPP displacing LN2 was captured in RTDs, flow rate, pressure, and liquid level plots. The LN2 level within the 200L CLV indicated that LN2 is circulating through the transfer lines after each discharge cycle. The closed loop portion of the quasi-closed loop system was captured with pressure readings within the 200L CLV. Lastly, the discharge(40s), refill (60s, 100s, 120s), and delay (15s) are the three parts exhibited in this experimental direct acting piston type pump.

\section{ACKNOWLEDGMENTS}

Funding for the DES Projects has been provided by the U.S. Department of Energy, the U.S. National Science Foundation, the Ministry of Science and Education of Spain, the Science and Technology Facilities Council of the United Kingdom, the Higher Education Funding Council for England, the National Center for Supercomputing Applications at the University of Illinois at Urbana-Champaign, the Kavli Institute of Cosmological Physics at the University of Chicago, Financiadora de Estudos e Projetos, Fundação Carlos Chagas Filho de Amparo à Pesquisa do Estado do Rio de Janeiro, Conselho Nacional de Desenvolvimento Científico e Tecnológico and the Ministério da Ciência e Tecnologia, the Deutsche Forschungsgemeinschaft and the Collaborating Institutions in the Dark Energy Survey.

The Collaborating Institutions are Argonne National Laboratories, the University of California at Santa Cruz, the University of Cambridge, Centro de Investigaciones Energeticas, Medioambientales y Tecnologicas-Madrid, the University of Chicago, University College London, DES-Brazil, Fermilab, the University of Edinburgh, the University of Illinois at Urbana-Champaign, the Institut de Ciencies de l'Espai (IEEC/CSIC), the Institut de Fisica d'Altes Energies, the Lawrence Berkeley National Laboratory, the Ludwig-Maximilians Universität and the associated Excellence Cluster Universe, the University of Michigan, the National Optical Astronomy Observatory, the University of Nottingham, the Ohio State University, the University of Pennsylvania, the University of Portsmouth, SLAC, Stanford University, the University of Sussex, and Texas A\&M University.

\section{REFERENCES}

1. M. Alvarez and H. Cease. "DECam LN2 Over-Pressure Pump Process Cylinder Testing Dec. 20-22, 2011," Dark Energy Document Database. 6106(2011).

2. M. Alvarez. "Lab A Over-Pressure Pump Test February Tests" Dark Energy Document Database. 6143 (2012).

3. M. Alvarez, (2012) The Development of Cryogenic Over-Pressure Pump. Unpublished MS Thesis. Illinois Institute of Technology and Fermi National Accelerator Laboratory. http://lss.fnal.gov/archive/masters/ fermilab-masters-2012-03.pdf

4. H. Cease and M. Alvarez. "DECam Over-Pressure Pump Cryo Safety Review Documentation." Dark Energy Document Database. 5787(2012).

5. H. Cease., D. Depoy, G. Derylo, H.T. Diehl, J. Estrada, B. Flaugher, K. Kuk, K. Kuhlmann, A. Lathrop, K. Shultz, R.J. Reinert, R.L. Schmitt, A. Stefanik, and A. Zhao. Cooling the Dark Energy Camera CCD array using a closed-loop, two-phase liquid nitrogen system. 2009: Fermilab Conference 10-242-PPD.

6. T.L. Henshaw, Reciprocating Pumps. New York, Van Nostrand Reinhold Company Inc. 1987.

7. The Dark Energy Survey. 2012. 16 April 2012. http://www. darkenergysurvey.org/index.shtml 